\title{
Pearson syndrome in a child transplanted for Diamond- Blackfan anemia
}

\author{
Vedat Uygun, M.D. ${ }^{a}$, Hayriye Daloğlu, M.D. ${ }^{a}$, Seda Öztürkmen, M.D. ${ }^{b}$, Gülsün Karasu, M.D. ${ }^{b}$ and \\ Akif Yeşilipek, M.D. ${ }^{b}$
}

\begin{abstract}
Pearson syndrome (PS), shares a number of overlapping features with Diamond-Blackfan anemia (DBA), including early onset of severe anemia, making differential diagnosis important. Differential diagnosis of DBA and PS is critical, since those with DBA may respond to treatment with steroids, may undergo remission, or may benefit from hematopoietic stem cell transplantation (HSCT). However, patients with PS have a different prognosis, with a very high risk of developing acidosis, metabolic problems, and pancreatic dysfunction, and a shorter life expectancy than those with DBA. Here we present a patient who underwent HSCT for DBA but was subsequently diagnosed with PS after developing some complications. Keywords: Diamond-Blackfan anemia, hematopoietic stem cell transplantation, Pearson syndrome.
\end{abstract}

http: / / dx.doi.org/10.5546/ aap.2021.eng.e559

To cite: Uygun V, Daloğlu H, Öztürkmen S, Karasu G, Yeşilipek A. Pearson syndrome in a child transplanted for Diamond-Blackfan anemia. Arch Argent Pediatr 2021;119(5):e559-e561.

\section{Acronyms and abbreviations}

PS: Pearson Syndrome.

DBA: Diamond-Blackfan anemia.

HSCT: Hematopoietic stem cell transplantation.

GVHD: Graft versus host disease.

CsA: Cyclosporine A.

NMA: Non-myeloablative.

a. İstinye University Faculty of Medicine, MedicalPark Antalya Hospital, Department of Pediatric Bone Marrow Transplantation Unit, Antalya, Turkey.

b. MedicalPark Antalya Hospital, Department of Pediatric Bone Marrow Transplantation Unit, Antalya, Turkey.

E-mail address:

Vedat Uygun, M.D.: veddat@hotmail.com

Funding: None.

Conflict of interest: None.

Received: 2-4-2021

Accepted: 3-31-2021

\section{INTRODUCTION}

Diamond-Blackfan anemia (DBA) is characterized by congenital severe hyporegenerative, macrocytic anemia and variably associated congenital malformations with growth retardation. Incidence is estimated to be seven cases for every million born children. ${ }^{1}$ Mutations in ribosomal protein (RP) genes are generally responsible for the disorder. ${ }^{2}$ The mainstays of therapy for DBA are corticosteroids and blood transfusion. Hematopoietic stem cell transplantation (HSCT) has been employed with success in steroid-refractory patients. ${ }^{3}$ Pearson syndrome (PS), a relatively rare condition (estimated incidence of 1 case / million newborns) ${ }^{4}$ caused by large deletions in mitochondrial DNA, presents in infancy with transfusion dependent severe macrocytic anemia often associated with variable degrees of pancytopenia, metabolic acidosis, and tissue dysfunction.

Differential diagnosis of DBA and PS is critical, since those with DBA may respond to treatment with steroids, may undergo remission, or may benefit from HSCT. However, patients with PS have a different prognosis, with a very high risk of developing acidosis, metabolic problems, and pancreatic dysfunction, and a shorter life expectancy than those with DBA. Steroids treatment is not indicated for PS, as it may increase the risk of complications without benefit, ${ }^{5}$ and HSCT is not among the primary treatments. $^{6}$

Here we present a patient who underwent HSCT for DBA but was subsequently diagnosed with PS after developing some complications.

\section{Case presentation}

The patient presented to a medical center at the age of 2 months due to severe pallor. Because of severe anemia with a hemoglobin level of $2.6 \mathrm{~g} / \mathrm{dl}$, bone marrow aspiration was performed, and DBA was diagnosed due to erythroid aplasia. Methylprednisolone $2 \mathrm{mg} / \mathrm{kg}$ was administered; however, it was discontinued after 4 months because of cataract development. Meanwhile, there was an opportunity to analyze the sequence 
of the patient's RPS19 gene which accounts about $25 \%$ of total DBA patients, ${ }^{7}$ and the result was reported to be normal. During follow-up, mild thrombocytopenia and mild neutropenia were noted. As the patient had a full-matched sibling donor (20 year old, male), the patient was admitted to our center at the age of 20 months for HSCT.

He was administered busulfan at a myeloablative dose in four days $(4.8 \mathrm{mg} / \mathrm{kg} /$ day), $150 \mathrm{mg} / \mathrm{m}^{2}$ fludarabine, $10 \mathrm{mg} / \mathrm{kg}$ thiotepa and $30 \mathrm{mg} / \mathrm{kg}$ anti-thymocyte globulin (ATGFresenius) as the conditioning regimen. He was transplanted with bone marrow stem cells with a dose of $10 \times 10^{8} / \mathrm{kg}$ total nucleated cells and $7 \times 10^{6} / \mathrm{kg} \mathrm{CD} 34(+)$ cells. Graft versus host disease (GVHD) prophylaxis was carried out with cyclosporine A (CsA), and $10 \mathrm{mg} / \mathrm{m}^{2}$ methotrexate on days 1,3 , and 6 .

The patient had no problems in the posttransplant period except for severe mucositis. Thrombocyte and neutrophil engraftments occurred at days 9 and 16, respectively. On the 24th day, the patient developed sepsis with hypoglycemia and hyperammonemia, and lactate increased to $10-12 \mathrm{mmol} / \mathrm{L}$. In the same period, the patient developed direct hyperbilirubinemia due to liver GVHD and it was decided to switch CsA to tacrolimus and to add $2 \mathrm{mg} / \mathrm{kg}$ methylprednisolone. Although his general condition gradually improved and cholestasis regressed, his lactate level remained elevated. The patient was considered to have metabolic disease. Therefore, it was decided to screen for PS, which is clinically similar to DBA. In the analysis of DNA separated before transplantation, a mitochondrial deletion indicating PS was found (heteroplasmic deletion with probe ratio 0.4, encompassing ATP6, COX3, ND3, ND4 and ND5 genes within the detection limits of Multiplex Ligation-Dependent Probe Amplification (MLPA)). In addition, bone marrow stains from the time of diagnosis were retrospectively reviewed, and rare vacuolization was observed in myeloid and erythroid precursors, which may support the diagnosis of PS. The patient was discharged on the 69th day for outpatient followup. However, because of some social problems, the patient was followed-up in another city and developed sepsis at 5 months post HSCT. Despite support, he worsened rapidly and died on the $167^{\text {th }}$ day of transplantation.

\section{DISCUSSION}

DBA and PS, which have common features, may not be distinguished from each other if there is no awareness of the disease similarities at the initial work-up, and a misdiagnosis might persist for years. An important finding that can be noted during differential diagnosis is vacuolization in myeloid and erythroid precursors in PS. When there were findings indicating PS in this case, we requested the initial bone marrow stains used in diagnosis and observed rare vacuolization in the myeloid and erythroid precursors. When the clinical and laboratory findings of the patient were retrospectively evaluated, it was determined that the vacuoles were previously interpreted as myelodysplasia, and that Pearson syndrome was not considered due to the absence of other clinical problems aside from anemia.

It can be argued that damage was caused by the transplant in this case, which was performed for the erroneous diagnosis of DBA. As far as we know, there are 4 reports of PS patients who have received HSCT, but only one of them survived. ${ }^{8-11}$ Interestingly, two of them underwent transplant with the diagnosis of DBA like our case but were diagnosed with PS during a research study performed after HSCT. Although no information was provided on the procedures applied for HSCT and follow-up of the patients, both patients were lost before the age of 3 . The other 2 patients engrafted and achieved normal blood counts. One of them underwent a second transplant due to rejection and developed a transient metabolic encephalopathy after a nonmyeloablative (NMA) conditioning regimen and GVHD prophylaxis with CsA. This patient developed several metabolic problems and acute myeloid leukemia 12 months post-HSCT and died. The second patient survived at 3 years follow-up after HSCT performed with an NMA regimen and infused cord blood as the stem cell source. GVHD prophylaxis was not specified in that report. Interestingly, HSCT was associated not only with improved hematopoiesis, but also with resolution of lactacidemia. ${ }^{10}$ Our patient underwent a myeloablative conditioning regimen without a major complication. Although it is not possible to point to an optimal conditioning regimen in PS due to the lack of cases, we can conclude that there is no major toxicity even in the myeloablative dose and no serious side effects with GVHD prophylaxis with CsA and methotrexate. 
Although HSCT is traditionally not recommended for the hematopoietic complications of PS because of their tendency to improve spontaneously, all of the patients underwent transplant simply due to their worsening hematological conditions. ${ }^{8-11}$ According to these limited cases, HSCT may correct hematological deterioration in PS. Evidence of the allogeneic HSCT benefits in inherited metabolic disorders raises the question of whether HSCT is of benefit for metabolic problems in PS. Among the reported patients, only one case showed improvement in metabolic problems. This case differed from the others in the use of cord blood as a stem cell source and in a significantly longer follow-up period. ${ }^{10}$ Cord blood is less mature than other stem cell sources, and its biological immaturity and possible regenerative effects might be the cause of the improvement of metabolic problems in the nonhematopoietic tissues of that patient. Further, this patient was followed for three years after HSCT, which is sufficient time for hematopoietic cells to correct the biochemical injury of metabolic problems in non-hematological organs. None of the other patients, including ours, survived long enough to see this benefit.

In patients presenting with congenital anemia, screening is recommended for DBA as well as PS, even if the general condition is good and there are no syndromic findings. Bone marrow transplantation may be a treatment option in PS, particularly in patients with worsening hematopoiesis. It remains essential to determine the correct diagnosis to assure appropriate followup and additional supportive treatment.

\section{REFERENCES}

1. Willig TN, Niemeyer CM, Leblanc $T$, Tiemann $C$, et al. Identification of new prognosis factors from the clinical and epidemiologic analysis of a registry of 229 Diamond-Blackfan anemia patients. DBA group of Societe d'Hematologie et d'Immunologie Pediatrique (SHIP), Gesellshaft fur Padiatrische Onkologie und Hamatologie $(\mathrm{GPOH})$, and the European Society for Pediatric Hematology and Immunology (ESPHI). Pediatr Res. 1999; 46(5):553-61.

2. Da Costa L, O'Donohue MF, van Dooijeweert B, AlbrechtK, et al. Molecular approaches to diagnose Diamond-Blackfan anemia: The EuroDBA experience. Eur J Med Genet. 2018; 61(11):664-73.

3. Fagioli F, Quarello P, Zecca M, Lanino E, et al. Haematopoietic stem cell transplantation for Diamond Blackfan anaemia: a report from the Italian Association of Paediatric Haematology and Oncology Registry. $\mathrm{Br}$ J Haematol. 2014; 165(5):673-81.

4. Farruggia P, Di Cataldo A, Pinto RM, Palmisani E, et al. Pearson Syndrome: A Retrospective Cohort Study from the Marrow Failure Study Group of A.I.E.O.P. (Associazione Italiana Emato-Oncologia Pediatrica). JIMD Rep. 2016; 26:37-43.

5. Finsterer J, Frank M. Glucocorticoids for mitochondrial disorders. Singapore Med J. 2015; 56(2):122-3.

6. Farruggia P, Di Marco F, Dufour C. Pearson syndrome. Expert Rev Hematol. 2018; 11(3):239-46.

7. Willig TN, Draptchinskaia N, Dianzani I, Ball S, et al. Mutations in ribosomal protein S19 gene and diamond blackfan anemia: wide variations in phenotypic expression. Blood. 1999; 94(12):4294-306.

8. Gagne KE, Ghazvinian R, Yuan D, Zon RL, et al. Pearson marrow pancreas syndrome in patients suspected to have Diamond-Blackfan anemia. Blood. 2014; 124(3):437-40.

9. Tumino M, Meli C, Farruggia P, La Spina M, et al. Clinical manifestations and management of four children with Pearson syndrome. Am J Med Genet A. 2011;155A(12):30636.

10. Hoyoux C, Dresse MF, Robinet S, Forget $\mathrm{P}$, et al. Cord blood transplantation in a child with Pearson's disease. Pediatr Blood Cancer. 2008; 51(4):566.

11. Faraci M, Cuzzubbo D, Micalizzi C, Lanino E, et al. Allogeneic bone marrow transplantation for Pearson's syndrome. Bone Marrow Transplant. 2007; 39(9):563-5. 